\title{
Evolution of phytochemical and antioxidant activity of Tunisian carob (Ceratonia siliqua L.) pods during maturation
}

\author{
Khadija Ben Othmen ${ }^{1}$, Walid Elfalleh ${ }^{2 *}$, Belgacem Lachiheb ${ }^{1}$ and Mansour Haddad ${ }^{1}$
}

\begin{abstract}
The Ceratonia siliqua fruits contain several substances known to have high adaptability to environmental conditions. The aim of this paper is to evaluate the changes in physicochemical properties of different Tunisian provenances of carob pulps harvested at three ripening stages. Furthermore, six provenances were investigated during the ripening process in terms of their moisture, ash, minerals, bioactive compounds, antiradical activity and sugar profile. The results demonstrated that all examined parameters are highly influenced by geographic origin. Concerning ripening impact, our data showed that water and ash content significantly decreased during the development of six provenances, as well as the bioactive and mineral contents. Thus, the total polyphenols (TP), total flavonoids (TF) and condensed tannins (CT) contents exhibited the highest levels in the unripe fruits. The antiradical activity trend was positively correlated to the behavior of the bioactive compounds content. Moreover, the sucrose, glucose and fructose were the main sugar qualified and quantified in carob pods at different ripening stages. At the maturity stage, the monosaccharide contents (glucose and fructose) were slightly reduced, while, the sucrose was rapidly accumulated. In conclusion, the ripening process diversely affected the nutritional composition and generally extended the exploitation of carob fruits. The study could provide valuable information about the suitability of carob pods at different maturity stages as potential biomaterials for nutraceutical applications.
\end{abstract}

Keywords: Ceratonia siliqua L., ripening, provenances, biochemical composition, anti-radical capacity

'Laboratoire d'Aridocultures et des cultures oasiennes, Institut des Régions Arides, Nahel, Gabès, Tunisia.

${ }^{2}$ Laboratoire Energie, Eau, Environnement et Procèdes, (LEEEP)LR18ES35, Ecole Nationale d'Ingénieurs de Gabès, Université de Gabès, Gabès, Tunisia.

${ }^{*}$ Corresponding author: W. Elfalleh E-mail: walid.elfalleh@fst.rnu.tn

DOI: 10.2478/ebtj-2019-0016

\section{Introduction}

Carob trees (Ceratonia siliqua L.), belong to the Caesalpinaceae sub family of the leguminosae family. For a long time, they have grown in Mediterranean areas $(1,2)$, including Maghreb countries in Nord-Africa, for forestry and consumption (3). They have been posteriorly planted in USA and Australia as an ornamental and shade tree (4). The carob fruit has been widely investigated due to the presence of various compounds as well as an important level of carbohydrates, minerals and phenolic compounds, which contribute to the great nutritive and functions potential $(1,3,5)$. Therefore, it has been mainly used in human nutrition, including syrups, popular drink and Arabic confectionery (1). In addition, as a feed for livestock (6) and especially used in traditional medicine to prevent or/and control health diseases (3). Besides, it has been qualified as an important raw material in food and non-food industries and afforded valuable products $(2,7)$, especially locust bean gum obtained from endosperm and marketed E410, which has been used as a stabilizer in emulsions $(1,8)$. Furthermore, the pods are used as cacao substitute(9), also as a raw material for alcohol and sugars production, in addition, it was applied in pharmaceutical, cosmetic and textile industries $(1,5,7)$.

Sugars and bioactive phenolic compounds are the main components of carob tree pulps (10). Indeed, pulps are considered as an interesting source of sugars which determine its organoleptic quality and its commercial value $(1,11)$. They present a significant concen- 
tration of bioactive principles that often reflect a considerable antioxidant potential (12) and confer an important medicinal and functional properties $(3,4)$. Besides, it is proven that the carob pulps contain a reasonable amount of mineral elements (2) which contribute a considerable nutritional value. In addition, it is known that geographical origin influences this biochemical composition. Indeed, the genetic and environmental expressions were known by its considerable effect on enzymatic activities that are involved in the biosynthesis and biodegradation of several primary and secondary metabolites (15). Therefore, pulps collected from Portugal (16) contain higher concentrations of total polyphenols than those collected from Algeria (12) and Tunisia (17). Besides, it has been also proved that the ripening process alters significantly this composition. Indeed, during the increasing of ripening level several primary and secondary metabolites are activated, then each compound will serve to an appropriate function to determine the final fruit characteristics. Accordingly, it was showed that the carob pods achieved the highest sucrose content at the ripe stage (11), consequently the industries producing the carob syrup, carob powder and bioethanol could to be interested in mature carob pods. However, in nutritional and functional term, the immature fruits are the preferred, since they contained the highest levels of phenolic content at the unripe stage (12).Therefore, it is necessary to study the biochemical variation of the carob pods harvested from different regions of Tunisia and evaluate its susceptibility to ripening process. This study aims to classify different carob provenance at different harvest dates based on their sugar content and bioactive phenolic compounds.

\section{Materials and Methods \\ Plant materials}

Fresh carob fruits were harvested in 2015 at different dates during ripening (06 June: unripe stage, 06 July: mid-ripe stage, 03 August: ripe stage), from different Tunisian areas, which were in the south, center and north of Tunisia (Table 1). The harvest dates were selected according to color change that accompanies the maturity. The seeds were manually removed after the fruit drying. The pulps were grounded to the fine powder, sieved through 100 mesh stainless steel sieves and stored at $4^{\circ} \mathrm{C}$ for further analysis.

\section{Determination of moisture}

Water content of tested provenances was determined by placing the pods in oven drying at 60 to 70 degrees until obtaining a stable weight (18).

\section{Determination of ash and minerals content}

The ash and minerals content of carob pods were assessed according to Oziyci et al. (2). One $\mathrm{g}$ from each dried carob powder was placed in a muffle furnace at $550^{\circ} \mathrm{C}$ for $6 \mathrm{~h}$ and the results were expressed as a percentage of dry weight. The minerals elements (iron, sodium, zinc, potassium and magnesium) of ashes samples were determined according to Elfalleh et al. (19). The cooled ashes were dissolved in $5 \mathrm{~mL}$ ultrapure water and $1 \mathrm{~mL}$ concentrated hydrochloric acid and were subjected to boiling. The obtained mixture was filtered, and the obtained filtrates were adjusted to $100 \mathrm{~mL}$ with ultrapure water and quantified by Atomic absorption spectroscopy (AAS).

\section{Determination of sugars profile}

The extraction process of carbohydrate from carob pods was performed using a reflux system as reported by Chaira et al. (20). Fructose, glucose and sucrose were measured by using an Agilent HPLC-RID System (HP. 1100) (21). The separation was performed on a ZORBAX column (Eclipse plus Column C18: $250 \mathrm{~mm} \times 4.6$ $\mathrm{mm}$ i.d., $5 \mu \mathrm{m}$ ). The detection was executed with RID-10 detector. The column temperature was maintained at $40^{\circ} \mathrm{C}$. The mobile phase was composed of $75 \%$ water and $25 \%$ acetonitrile at a flow rate of $1.5 \mathrm{~mL} / \mathrm{min}$. The concentration was determined by applied the external standards methods. The standards solutions (fructose, glucose, sucrose and maltose) were prepared in acetonitrile/water and used for calibration curves (at different concentrations within the range of $25-300 \mu \mathrm{g} / \mathrm{mL}$. The peck surface was calculated by using Shimadzu LabSolutions software version.5.42.

\section{Determination of bioactive compounds Extract preparation}

The extraction procedure of phenolic compounds was carried out using conventional system. Carob powders were subjected to cold maceration using water solvent. $10 \mathrm{~g}$ of each sample were homogenized in $100 \mathrm{~mL}$ of distilled water and shaken for $90 \mathrm{~min}$ in darkness. The obtained extracts were centrifuged twice (5000 $\mathrm{rpm}, 10 \mathrm{~min}$ ) and stored at $4^{\circ} \mathrm{C}$ for further uses.

Table 1. Geographic data of carob tree provenances

\begin{tabular}{|l|l|l|l|l|}
\hline Code & Origin & Longitude & Latitude & Bioclimatic zone \\
\hline SK & Skhira & $34^{\circ} 18^{\prime} 32.82^{\prime \prime} \mathrm{N}$ & $10^{\circ} 02^{\prime} 57.63^{\prime \prime} \mathrm{E}$ & Arid with soft winter \\
\hline KE & Ksour Essef & $35^{\circ} 25^{\prime} 03.26^{\prime \prime} \mathrm{N}$ & $10^{\circ} 59^{\prime} 51.57^{\prime \prime} \mathrm{E}$ & Semi-arid with soft winter \\
\hline $\mathrm{MN}$ & Monastir & $35^{\circ} 39^{\prime} 55.61^{\prime \prime} \mathrm{N}$ & $10^{\circ} 51^{\prime} 33.33^{\prime \prime} \mathrm{E}$ & Semi-arid with soft winter \\
\hline TN & Tunis & $36^{\circ} 50^{\prime} 11.93^{\prime \prime} \mathrm{N}$ & $10^{\circ} 11^{\prime} 41.34^{\prime \prime} \mathrm{E}$ & Semi-arid with soft winter \\
\hline ZG & Zaghouan & $36^{\circ} 24^{\prime} 32.83^{\prime \prime} \mathrm{N}$ & $10^{\circ} 08^{\prime} 32.83^{\prime \prime} \mathrm{E}$ & Arid with soft winter \\
\hline GB & Gabes & $33^{\circ} 52^{\prime} 49.29^{\prime \prime} \mathrm{N}$ & $10^{\circ} 05^{\prime} 26.80^{\prime \prime} \mathrm{E}$ & Arid with soft winter \\
\hline
\end{tabular}


Table 2. Variation of moisture (\%) and ash (\%) content of carob pods provenances during ripening process

\begin{tabular}{|c|c|c|c|c|c|c|}
\multicolumn{3}{|c}{ Water content $\%)$} & \multicolumn{2}{c}{ Ash (\%) } \\
\hline code & Unripe & Mid-ripe & Ripe & Unripe & Mid-ripe \\
\hline SK & $63.31 \pm 1.11^{\mathrm{Ca}}$ & $51.47 \pm 2.17^{\mathrm{Ba}}$ & $9.03 \pm 2.13^{\mathrm{Aa}}$ & $4.27 \pm 0.26^{\mathrm{Bcb}}$ & $3.96 \pm 0.03^{\mathrm{Bc}}$ & $3.22 \pm 0.09^{\mathrm{Ab}}$ \\
\hline KE & $76.65 \pm 1.13^{\mathrm{Ce}}$ & $62.01 \pm 2.19^{\mathrm{Bc}}$ & $13.73 \pm 3.22^{\mathrm{Ac}}$ & $3.30 \pm 0.14^{\mathrm{Ba}}$ & $3.22 \pm 0.04^{\mathrm{Bb}}$ & $2.72 \pm 0.05^{\mathrm{Aa}}$ \\
\hline MN & $70.16 \pm 3.15^{\mathrm{Cc}}$ & $60.20 \pm 3.21^{\mathrm{Bbc}}$ & $11.54 \pm 2.45^{\mathrm{Ab}}$ & $3.81 \pm 0.17^{\mathrm{Bb}}$ & $2.64 \pm 0.06^{\mathrm{Aa}}$ & $2.70 \pm 0.06^{\mathrm{Aa}}$ \\
\hline TN & $76.56 \pm 1.09^{\mathrm{Ce}}$ & $62.93 \pm 1.75^{\mathrm{Bc}}$ & $14.27 \pm 2.17^{\mathrm{Ac}}$ & $4.97 \pm 0.05^{\mathrm{Cde}}$ & $4.75 \pm 0.06^{\mathrm{Bd}}$ & $3.45 \pm 0.06^{\mathrm{Ab}}$ \\
\hline ZG & $72.79 \pm 1.05^{\mathrm{Cd}}$ & $58.34 \pm 3.17^{\mathrm{Bbc}}$ & $11.30 \pm 2.45^{\mathrm{Ab}}$ & $4.49 \pm 0.25^{\mathrm{Bcd}}$ & $3.01 \pm 0.04^{\mathrm{Ab}}$ & $2.51 \pm 0.31^{\mathrm{Aa}}$ \\
\hline GB & $68.67 \pm 2.10^{\mathrm{Cb}}$ & $56.14 \pm 1.5^{\mathrm{Bab}}$ & $9.38 \pm 1.10^{\mathrm{Aa}}$ & $5.16 \pm 0.11^{\mathrm{Be}}$ & $4.71 \pm 0.22^{\mathrm{Ad}}$ & $4.58 \pm 0.15^{\mathrm{Ac}}$
\end{tabular}

Results are expressed as the mean \pm SD $(n=3)$. Different capital letters represent significant variation $(p<0.05)$ between ripening stages; different lowercase letters represent significant difference variation $(p<0.05)$ between provenances according to Tukey test.

\section{Total polyphenols content}

Total polyphenols (TP) content was evaluated in obtained extracts by using the Folin-Ciocalleu colorimetric assays as described by Assadi et al. (21). Each prepared extract $(100 \mu \mathrm{L})$ was mixed with concentrated Folin- Ciocalteu solution (500 $\mu \mathrm{L})$ and sodium carbonate solution $(4 \mathrm{~mL}, 1 \mathrm{M})$. The absorbance was measured at $765 \mathrm{~nm}$ by means of a UV-visible spectrophotometer (T60 UV-visible spectrophotometer) after incubation for $90 \mathrm{~min}$ in dark condition. Gallic acid was used as a standard and results were expressed as gram Gallic acid equivalent per $100 \mathrm{~g}$ of dry weight (g GAE/ $100 \mathrm{~g}$ DW).

\section{Total flavonoids content}

Total flavonoids content was investigated by using spectrophotometrically method according to Bahorun et al.(22). One milliliter of each extract was homogenized with one milliliter of $10 \%$ aluminum trichloride solution. The obtained mixtures were incubated for $30 \mathrm{~min}$ at room temperature for immediate absorbance, which was carried out by using a UV-visible spectrophotometer (T60 UV-visible spectrophotometer) at $430 \mathrm{~nm}$. Rutin was used as a standard and the results were expressed as gram rutin equivalent per $100 \mathrm{~g}$ of dry weight ( $\mathrm{g}$ RE/ $100 \mathrm{~g}$ DW).

\section{Condensed tannins content}

The condensed tannin content of obtained extracts was determined using colorimetric methods demonstrated by Khali et al. (23). Concentrated hydrochloric acid $(750 \mu \mathrm{L})$ was mixed with $1.5 \mathrm{~mL}$ of vanillin solution (4\%) prepared in methanol and $250 \mu \mathrm{L}$ of each sample. The obtained aliquots were incubated at room temperature for $15 \mathrm{~min}$. The absorbance was measured at $500 \mathrm{~nm}$ using a UV-visible spectrophotometer (T60 UV-visible spectrophotometer). Catechin was used as a standard and results were expressed as gram catechin equivalent per $100 \mathrm{~g}$ of dry weight (g CE/ 100 g DW).

\section{Antioxidant activity}

The Antioxidant activity of prepared extracts was measured in terms of their capacity to trap the free radical by using DPPH assay as described by Benchikh et al. (12). DPPH solution (0.2
$\mathrm{mM}$ ) previously prepared in methanol was mixed with $25 \mu \mathrm{L}$ of each extract and slowly shacked. The obtained samples were incubated for $30 \mathrm{~min}$ in dark condition before being measured at $517 \mathrm{~nm}$ by using a visible a UV-visible spectrophotometer (T60 UV-visible spectrophotometer). Trolox was used as a standard and results were expressed as Trolox equivalent (TE) per $100 \mathrm{~g}$ of dry weight (g TE/ $100 \mathrm{~g}$ DW).

\section{Statistical analysis}

Statistical analyses were carried out using Xlstat software Ver. 2017 (www.xlstat.com). Data were subjected to one-way analysis of variance (ANOVA) Values are presented as a mean \pm standard deviation (SD).

\section{Results}

\section{Water and ash contents}

The variation of moisture and ash content during repining were determined and the results were expressed as a percentage (Table 2). The statistical data showed that the mentioned parameters were significantly influenced by the ripening stage and geographic origin. The both values were higher at the unripe stage, less important at the mid-ripe and lower at the ripe one. The decline pattern of moisture was acute, whereas, that of ash was slight. The water content varied from $76.65 \pm 1.13 \%$ to $9.03 \pm 2.13 \%$ and the ash content ranged from $5.16 \pm 0.11 \%$ to $2.51 \pm 0.31 \%$ at different ripening stages.

\section{Minerals compounds content}

The statistical comparison (Table 3 ) showed a significant quantitative variability based on ripening stages and geographic origin. Potassium, sodium, magnesium, iron and zinc are the main minerals detected throughout maturity of studied pods. Again, the quantitative variation exhibited an uniform evolutionary pattern. In fact, the amounts of minerals compounds were statistically more important at unripe stage than those obtained at the mid-ripe and ripe ones, except for $\mathrm{Mg}$ and $\mathrm{Zn}$ levels which were not affected by ripening. In the same way, a quantitative deviation was recorded between provenances, however, all tested samples were characterized by an interest- 
Table 3. Changes in minerals compounds content of carob provenances during ripening process

\begin{tabular}{|c|c|c|c|c|c|c|c|}
\hline $\begin{array}{l}\text { Mineral } \\
\text { compound }\end{array}$ & Stage & SK & KE & $\mathrm{MN}$ & $\mathrm{TN}$ & ZG & GB \\
\hline \multirow{3}{*}{$\mathrm{Fe}(\mu \mathrm{mol} / \mathrm{g} \mathrm{DW})$} & Unripe & $0,40 \pm 0,00^{c c}$ & $0,21 \pm 0,00^{\mathrm{Bb}}$ & $0,37 \pm 0,00^{c c}$ & $0,25 \pm 0,00^{\mathrm{Bb}}$ & $0,17 \pm 0,00^{\text {ca }}$ & $0,91 \pm 0,00^{c d}$ \\
\hline & Mid-ripe & $0,15 \pm 0,00^{\mathrm{Bb}}$ & $0,14 \pm 0,00^{A b}$ & $0,21 \pm 0,00^{B C}$ & $0,15 \pm 0,00^{A b}$ & $0,09 \pm 0,00^{B a}$ & $0,18 \pm 0,00^{B C}$ \\
\hline & Ripe & $0,14 \pm 0,00^{\mathrm{Ac}}$ & $0,14 \pm 0,00^{A c}$ & $0,18 \pm 0,00^{\mathrm{Ad}}$ & $0,15 \pm 0,00^{\mathrm{Ac}}$ & $0,06 \pm 0,00^{\mathrm{Aa}}$ & $0,095 \pm 0,00^{A b}$ \\
\hline \multirow{3}{*}{$\mathrm{Na}(\mu \mathrm{mol} / \mathrm{g} \mathrm{DW})$} & Unripe & $4,56 \pm 0,05^{\mathrm{ca}}$ & $7,27 \pm 0,02^{\mathrm{Cd}}$ & $5,44 \pm 0,00^{\mathrm{cb}}$ & $4,55 \pm 0,03^{\mathrm{Ca}}$ & $6,24 \pm 0,03^{c c}$ & $7,18 \pm 0,03^{\mathrm{Bd}}$ \\
\hline & Mid-ripe & $4,01 \pm 0,04^{\mathrm{Bb}}$ & $6,67 \pm 0,03^{\mathrm{Bd}}$ & $5,00 \pm 0,87^{B c}$ & $3,56 \pm 0,04^{\mathrm{Ba}}$ & $5,29 \pm 0,08^{B C}$ & $7,26 \pm 0,01^{\mathrm{Ae}}$ \\
\hline & Ripe & $3,72 \pm 0,00^{A b}$ & $6,04 \pm 0,87^{\mathrm{Ad}}$ & $3,96 \pm 0,89^{A b}$ & $1,94 \pm 0,03^{\text {Аа }}$ & $4,86 \pm 0,09^{A c}$ & $7,59 \pm 0,19^{\mathrm{Ae}}$ \\
\hline \multirow{3}{*}{$\mathrm{Zn}(\mu \mathrm{mol} / \mathrm{g} \mathrm{DW})$} & Unripe & $0,032 \pm 0,06^{\mathrm{Aa}}$ & $0,05 \pm 0,00^{\mathrm{Aa}}$ & $0,028 \pm 0,00^{\text {Aa }}$ & $0,06 \pm 0,00^{\mathrm{Aa}}$ & $0,05 \pm 0,00^{\text {Аa }}$ & $0,04 \pm 0,00^{\mathrm{Aa}}$ \\
\hline & Mid-ripe & $0,04 \pm 0,09^{\text {Aa }}$ & $0,05 \pm 0,00^{\mathrm{Aa}}$ & $0,014 \pm 0,00^{\text {Aa }}$ & $0,04 \pm 0,00^{\text {Аа }}$ & $0,05 \pm 0,00^{\text {Aa }}$ & $0,04 \pm 0,00^{\mathrm{Aa}}$ \\
\hline & Ripe & $0,05 \pm 0,00^{\text {Аa }}$ & $0,05 \pm 0,00^{\mathrm{Aa}}$ & $0,01 \pm 0,00^{\mathrm{Aa}}$ & $0,03 \pm 0,00^{\text {Aa }}$ & $0,01 \pm 0,00^{\mathrm{Aa}}$ & $0,02 \pm 0,00^{\mathrm{Aa}}$ \\
\hline \multirow{3}{*}{$\mathrm{K}(\mu \mathrm{mol} / \mathrm{g} \mathrm{DW})$} & Unripe & $132,35 \pm 0,36^{\mathrm{cd}}$ & $94,38 \pm 0,09^{\mathrm{cb}}$ & $80,47 \pm 0,62^{\mathrm{Ca}}$ & $174,25 \pm 15,5^{\mathrm{Ce}}$ & $114,6 \pm 0,21^{\mathrm{cc}}$ & $164,14 \pm 0,5^{\mathrm{Ce}}$ \\
\hline & Mid-ripe & $114,72 \pm 2,26^{\mathrm{Bd}}$ & $83,27 \pm 0,66^{\mathrm{BC}}$ & $53,79 \pm 0,06^{\mathrm{Ba}}$ & $120,93 \pm 6,20^{\mathrm{Be}}$ & $68,64 \pm 0,39^{\mathrm{Bb}}$ & $162,88 \pm 0,38^{B f}$ \\
\hline & Ripe & $68,15 \pm 0,45^{A c}$ & $46,19 \pm 0,92^{\text {Аa }}$ & $44,46 \pm 0,83^{\mathrm{Aa}}$ & $99,25 \pm 7,39^{\mathrm{Ad}}$ & $58,87 \pm 0,43^{A b}$ & $157,93 \pm 0,37^{\mathrm{Ae}}$ \\
\hline \multirow{3}{*}{$\mathrm{Mg}(\mu \mathrm{mol} / \mathrm{g} \mathrm{DW})$} & Unripe & $6,17 \pm 1,43^{\text {Аа }}$ & $6,54 \pm 0,94^{\text {Аа }}$ & $3,65 \pm 1,23^{\text {Аа }}$ & $7,91 \pm 0,4^{\text {Аa }}$ & $7,06 \pm 1,07^{\text {Aa }}$ & $4,89 \pm 0,92^{\mathrm{Aa}}$ \\
\hline & Mid-ripe & $6,07 \pm 1,25^{\text {Aa }}$ & $6,44 \pm 1,23^{\text {Аa }}$ & $1,81 \pm 0,37^{\text {Аа }}$ & $4,94 \pm 0,03^{\text {Аа }}$ & $6,31 \pm 1,22^{\mathrm{Aa}}$ & $4,86 \pm 0,57^{\mathrm{Aa}}$ \\
\hline & Ripe & $4,39 \pm 0,84^{\mathrm{Aa}}$ & $6,25 \pm 0,97^{\text {Аa }}$ & $1,38 \pm 0,19^{\text {Аа }}$ & $4,15 \pm 0,4^{\mathrm{Aa}}$ & $1,25 \pm 0,72^{\mathrm{Aa}}$ & $2,8 \pm 0,09^{\mathrm{Aa}}$ \\
\hline
\end{tabular}

Results are expressed as the mean \pm SD $(n=3)$. Fe, iron; Na, sodium; Zn, zind; K, potassium; Mg, magnesium; DW, dry weight. Different capital letters represent significant variation $(p<0.05)$ between ripening stages; different lowercase letters represent significant difference variation $(p<0.05)$ between provenances according to Tukey test.

ing yield of potassium, the sodium $(\mathrm{Na})$ and magnesium $(\mathrm{Mg})$ were present also in important amounts, while, iron $(\mathrm{Fe})$ and zinc $(\mathrm{Zn})$ detected as trace elements.

\section{Sugar profile}

The sucrose, fructose and glucose contents in carob pods at three different ripening stages, harvested from different $\mathrm{Tu}$ - nisian regions were shown in Table 4 . The results revealed that the geographical origin and ripening stages affects sugars yield in quantitative term but not in qualitative one. Indeed, the main sugars identified and quantified in carob pods during pods development, were sucrose, fructose and glucose. In addition, throughout maturity this variation was expressed by a slight reduction of fructose and glucose content and an

Table 4. Sugars profile of carob pods provenances influenced by ripening process

\begin{tabular}{|c|c|c|c|c|c|c|c|}
\hline Carbohydrates & Stages & SK & KE & $\mathrm{MN}$ & $\mathrm{TN}$ & ZG & GB \\
\hline \multirow{3}{*}{$\begin{array}{l}\text { Fructose ( } \mu \mathrm{mol} \\
\text { fructose/g DW) }\end{array}$} & Unripe & $1386.41 \pm 4.16^{\mathrm{cd}}$ & $1355.62 \pm 4.16^{\mathrm{cc}}$ & $1196.67 \pm 0.00^{\mathrm{Ca}}$ & $1304.85 \pm 6.66^{\mathrm{cb}}$ & $1463.8 \pm 10.81^{\mathrm{Ce}}$ & $1461.3 \pm 4.16^{\mathrm{ce}}$ \\
\hline & Mid-ripe & $1340.64 \pm 4.16^{\mathrm{Bf}}$ & $1046.88 \pm 4.99^{\mathrm{Ba}}$ & $1086.82 \pm 0.00^{\mathrm{Bb}}$ & $1126.77 \pm 1.66^{\mathrm{BC}}$ & $1150.07 \pm 2.50^{\mathrm{Bd}}$ & $1252.43 \pm 7.49^{\mathrm{Be}}$ \\
\hline & Ripe & $1118.45 \pm 1.66^{\mathrm{Ac}}$ & $989.46 \pm 5.83^{\mathrm{Aa}}$ & $980.3 \pm 0.00^{\mathrm{Aa}}$ & $1066.02 \pm 2.50^{\mathrm{Ab}}$ & $1090.98 \pm 3.33^{\mathrm{Ac}}$ & $1061.03 \pm 7.49^{A b}$ \\
\hline \multirow{3}{*}{$\begin{array}{l}\text { Glucose ( } \mu \text { mol } \\
\text { glucose/g DW) }\end{array}$} & Unripe & $694.88 \pm 0.00^{\mathrm{cd}}$ & $548.42 \pm 19.14^{c c}$ & $520.95 \pm 3.33^{c c}$ & $486.00 \pm 3.33^{\mathrm{cb}}$ & $532.61 \pm 0.83^{\mathrm{cc}}$ & $376.15 \pm 0.83^{\mathrm{ca}}$ \\
\hline & Mid-ripe & $314.57 \pm 0.83^{\mathrm{Ba}}$ & $436.07 \pm 0.00^{B d}$ & $348.69 \pm 2.50^{B b}$ & $484.34 \pm 7.49^{\mathrm{Be}}$ & $401.12 \pm 1.66^{\mathrm{BC}}$ & $352.85 \pm 1.66^{\mathrm{Bb}}$ \\
\hline & Ripe & $297.09 \pm 3.33^{\mathrm{Ab}}$ & $307.08 \pm 2.50^{\mathrm{Ab}}$ & $309.58 \pm 0.83^{\mathrm{Ab}}$ & $270.46 \pm 3.33^{\mathrm{Aa}}$ & $366.17 \pm 1.66^{A C}$ & $281.28 \pm 4.16^{\mathrm{Aa}}$ \\
\hline \multirow{3}{*}{$\begin{array}{l}\text { Sucrose }(\mu \mathrm{mol} \\
\text { sucrose/g DW) }\end{array}$} & Unripe & $299.15 \pm 1.75^{\mathrm{Ab}}$ & $444.13 \pm 4.38^{\mathrm{Ae}}$ & $481.79 \pm 1.75^{\mathrm{Af}}$ & $349.08 \pm 0.88^{\mathrm{Ac}}$ & $422.66 \pm 4.38^{\mathrm{Ad}}$ & $242.65 \pm 6.57^{\mathrm{Aa}}$ \\
\hline & Mid-ripe & $414.78 \pm 5.26^{\text {ва }}$ & $2064.7 \pm 2.19^{\text {Bd }}$ & $2525.91 \pm 0.44^{B f}$ & $685.46 \pm 3.94^{\mathrm{Bb}}$ & $2461.52 \pm 3.07^{\mathrm{Be}}$ & $1841.32 \pm 0.88^{B C}$ \\
\hline & Ripe & $2256.1 \pm 1.31^{\mathrm{cc}}$ & $3097.49 \pm 4.82^{\mathrm{cf}}$ & $2592.04 \pm 0.88^{\mathrm{cd}}$ & $2095.8 \pm 3.50^{\mathrm{cb}}$ & $2881.99 \pm 6.57^{\mathrm{Ce}}$ & $2035.79 \pm 1.75^{\mathrm{Ca}}$ \\
\hline
\end{tabular}

Results are expressed as the mean $\pm S D(n=3)$. Different capital letters represent significant variation $(p<0.05)$ between ripening stages; different lowercase letters represent significant difference variation $(p<0.05)$ between provenances according to Tukey test. 
obvious accumulation of sucrose. At the end of maturity all the provenances reached the highest sucrose amounts, which recorded significant difference between them. Indeed, the highest amount of sucrose was achieved by "KE" provenance $(3097 \pm 4.82 \mu \mathrm{mol} / \mathrm{g} \mathrm{DW})$ and the lowest is attained by "GB" provenance $(2035,79 \pm 1,75 \mu \mathrm{mol} / \mathrm{g} \mathrm{DW})$. Furthermore, our samples exhibited variation in the evolutionary behavior of sucrose. In fact, the provenances "SK" and "TN" showed two evolutional phases: a slight increase (from unripe to mid-ripe stage), followed by a speedy increment (from mid-ripe to ripe stage), while, the other provenances revealed a brutal increase followed by a slight raise.

\section{Extraction yield and polyphenols content}

The yields (\%) of dry aqueous extracts obtained from different provenances at different ripening stages were calculated and represented in Table 5. At the unripe stage, the whole provenances exhibited the lowest yield which ranged from $20.40 \pm 0.42 \%$ to $15.40 \pm 0.71 \%$. Then, a gradual increase was showed until reaching the highest yield at the end of maturity, these values varied from $63.20 \pm 0.71 \%$ to $50.60 \pm 0.57 \%$.

The total polyphenols, flavonoids and condensed tannins concentrations in carob pods were investigated at three ripening stages (Table 5). The statistical data showed that all constituents varied considerably according to the harvest date and the geographic origin. However, their content obtained from different provenances revealed an uniform progressive behavior. Thus, the highest levels were reached in the unripe stage and then decreased gradually to attain the lowest levels at the end of the maturity. An important variation was revealed between phenolics compounds contents of different provenances under the same stage. Thus, in the unripe stage, the provenance "ZG" showed the highest levels of total polyphenols (13.46 \pm $0.06 \mathrm{~g}$ EAG / $100 \mathrm{~g}$ MS) and condensed tannins $(6.67 \pm 0.05$ g EC / 100 g MS), while, its lowest amounts were reached by "TN". Concerning the flavonoids content, the immature pods obtained from "GB" provenance recorded the highest amount (562.91 $\pm 8.20 \mathrm{~g}$ ER / $100 \mathrm{~g} \mathrm{MS}$ ), whereas, at the same ripening

Table 5. Changes in bioactive compounds content of carob pods provenances during ripening process

\begin{tabular}{|c|c|c|c|c|c|c|c|}
\hline Parameters & Stages & SK & KE & $\mathrm{MN}$ & TN & ZG & GB \\
\hline \multirow{3}{*}{ Yield (\%) } & Unripe & $20.40 \pm 0.42^{A c}$ & $15.40 \pm 0.71^{\mathrm{Aa}}$ & $19.80 \pm 0.85^{A C}$ & $17.50 \pm 0.64^{\mathrm{Aab}}$ & $16.40 \pm 0.14^{\mathrm{Aa}}$ & $19.50 \pm 0.49^{\mathrm{Abc}}$ \\
\hline & Mid-ripe & $35.80 \pm 0.99^{\mathrm{Ba}}$ & $52.20 \pm 0.57^{\mathrm{BC}}$ & $52.00 \pm 0.28^{\mathrm{BC}}$ & $37.90 \pm 0.21^{\mathrm{Ab}}$ & $57.20 \pm 0.42^{\mathrm{Bd}}$ & $50.70 \pm 0.35^{B c}$ \\
\hline & Ripe & $53.90 \pm 0.35^{\mathrm{Cabc}}$ & $63.20 \pm 0.71^{c d}$ & $54.70 \pm 1.91^{\mathrm{cb}}$ & $50.60 \pm 0.57^{\mathrm{Ba}}$ & $56.80 \pm 0.14^{\mathrm{cc}}$ & $52.70 \pm 0.35^{\mathrm{cab}}$ \\
\hline \multirow{3}{*}{$\begin{array}{l}\text { Total polyphenols } \\
\text { (TP) } \\
\text { (g GAE/ } 100 \text { g DW) }\end{array}$} & Unripe & $12.91 \pm 0.11^{\mathrm{Ce}}$ & $12.11 \pm 0.66^{\mathrm{cd}}$ & $10.96 \pm 0.09^{\mathrm{cc}}$ & $4.57 \pm 0.12^{\mathrm{Ba}}$ & $13.46 \pm 0.06^{\mathrm{Ce}}$ & $10.14 \pm 0.10^{\mathrm{cb}}$ \\
\hline & Mid-ripe & $5.33 \pm 0.01^{\text {Bed }}$ & $4.25 \pm 0.02^{\mathrm{Bb}}$ & $4.66 \pm 0.05^{\mathrm{Bbc}}$ & $3.58 \pm 0.08^{\mathrm{Aa}}$ & $5.09 \pm 0.11^{\mathrm{Bcd}}$ & $5.67 \pm 0.07^{\mathrm{Be}}$ \\
\hline & Ripe & $2.72 \pm 0.10^{\mathrm{Aa}}$ & $3.24 \pm 0.23^{\mathrm{Ab}}$ & $3.22 \pm 0.24^{\mathrm{Ab}}$ & $3.11 \pm 0.12^{\mathrm{Ab}}$ & $3.09 \pm 0.11^{\mathrm{Aab}}$ & $4.92 \pm 007^{A C}$ \\
\hline \multirow{3}{*}{$\begin{array}{l}\text { Total flavonoids } \\
\text { (TF) } \\
\text { (mg RE/ } 100 \text { g DW) }\end{array}$} & Unripe & $537.78 \pm 1.07^{\mathrm{cbc}}$ & $522.65 \pm 5.51^{\mathrm{Bb}}$ & $560.80 \pm 2.71^{\mathrm{cbc}}$ & $275.91 \pm 2.54^{\text {Ca }}$ & $528.26 \pm 3.52^{\mathrm{cbc}}$ & $562.91 \pm 8.20^{B C}$ \\
\hline & Mid-ripe & $184.32 \pm 0.46^{\mathrm{Bd}}$ & $124.80 \pm 0.03^{A b}$ & $204.87 \pm 1.47^{\mathrm{Be}}$ & $103.01 \pm 0.61^{\mathrm{Ba}}$ & $167.97 \pm 0.54^{\mathrm{BC}}$ & $254.37 \pm 2.12^{\mathrm{Af}}$ \\
\hline & Ripe & $68.14 \pm 0.21^{\mathrm{Aa}}$ & $117.89 \pm 3.71^{\mathrm{Ab}}$ & $174.06 \pm 1.44^{\mathrm{Ac}}$ & $62.75 \pm 0.16^{\mathrm{Aa}}$ & $121.36 \pm 0.49^{\mathrm{Ab}}$ & $220.93 \pm 0.45^{\mathrm{Ad}}$ \\
\hline \multirow{3}{*}{$\begin{array}{l}\text { Condensed tannins } \\
\text { (CT) } \\
\text { (g CE/100 g DW) }\end{array}$} & Unripe & $5.96 \pm 0.06^{\mathrm{cd}}$ & $4.75 \pm 0.05^{c c}$ & $1.18 \pm 0.03^{\mathrm{Ba}}$ & $1.27 \pm 0.04^{\mathrm{Ba}}$ & $6.67 \pm 0.05^{\mathrm{Ce}}$ & $3.31 \pm 0.02^{\mathrm{cb}}$ \\
\hline & Mid-ripe & $1.52 \pm 0.01^{\mathrm{Be}}$ & $0.84 \pm 0.01^{\mathrm{Bb}}$ & $0.45 \pm 0.01^{\mathrm{Aa}}$ & $0.49 \pm 0.01^{\mathrm{Aa}}$ & $1.13 \pm 0.01^{B C}$ & $1.32 \pm 0.01^{\mathrm{Bd}}$ \\
\hline & Ripe & $0.30 \pm 0.00^{\mathrm{Aa}}$ & $0.33 \pm 0.00^{\mathrm{Aab}}$ & $0.38 \pm 0.00^{\mathrm{Ab}}$ & $0.38 \pm 0.00^{A b}$ & $0.58 \pm 0.01^{A c}$ & $0.58 \pm 0.01^{A c}$ \\
\hline
\end{tabular}

Results are expressed as the mean $\pm S D(n=3)$. DW, dry weight; GAE, gallic acid equivalent; RE, rutin equivalent; $C E$, catechin equivalent. Different capital letters represent significantvariation $(p<0.05)$ between ripening stages; different lowercase letters represent significant difference variation $(p<0.05)$ between provenances according to Tukey test.

Table 6. Changes in antiradical activity of carob pods provenances during ripening process

\begin{tabular}{|l|l|l|l|l|l|l|l|}
\hline Antiradical activity & stages & \multicolumn{1}{|c|}{ SK } & KE & MN & TN & ZG & GB \\
\hline & Unripe & $30.39 \pm 0.45^{\mathrm{Cd}}$ & $29.57 \pm 0.39^{\mathrm{cc}}$ & $26.18 \pm 0.78^{\mathrm{Cb}}$ & $20.98 \pm 0.15^{\mathrm{Ca}}$ & $32.78 \pm 0.78^{\mathrm{Ce}}$ & $25.74 \pm 0.18^{\mathrm{cb}}$ \\
\hline $\begin{array}{l}\text { DPPH } \\
\text { (g TE/ } 100 \mathrm{~g} \mathrm{DW})\end{array}$ & Mid-ripe & $14.29 \pm 0.59^{\mathrm{Bd}}$ & $12.67 \pm 0.48^{\mathrm{Bb}}$ & $13.56 \pm 0.66^{\mathrm{BC}}$ & $9.36 \pm 0.64^{\mathrm{Ba}}$ & $14.98 \pm 0.16^{\mathrm{Bd}}$ & $16.93 \pm 0.24^{\mathrm{Be}}$ \\
\hline & Ripe & $5.59 \pm 0.38^{\mathrm{Ab}}$ & $9.46 \pm 0.41^{\mathrm{Ac}}$ & $10.29 \pm 0.26^{\mathrm{Ad}}$ & $4.56 \pm 0.45^{\mathrm{Aa}}$ & $9.76 \pm 0.47^{\mathrm{Ac}}$ & $11.47 \pm 0.48^{\mathrm{Ae}}$ \\
\hline
\end{tabular}

Results are expressed as the mean $\pm S D(n=3)$. DW, dry weight; $T E$, Trolox equivalent antioxidant capacity. Different capital letters represent significant variation $(p<0.05)$ between ripening stages; different lowercase letters represent significant difference variation $(p<0.05)$ between provenances according to Tukey test. 
level, the lowest content was seen in "TN" provenance (275.91 $\pm 2.54 \mathrm{~g} \mathrm{ER} / 100 \mathrm{~g}$ MS).

\section{Antioxidant activity}

The DPPH free radical-scavenging activity was studied during development of carob fruits and the results were represented in Table 6. The data showed that the antioxidant level was influenced by ripening stage. However, it was the highest in immature fruits, intermediate in semi-mature and the lowest in mature ones. The antioxidant activity level was also origin-dependent, indeed, at the beginning of maturity, it exhibited the highest levels which ranged from $32.78 \pm 0.78 \mathrm{~g} \mathrm{TE} / 100 \mathrm{~g}$ DW(“ZG”) to $20.98 \pm 0.15 \mathrm{~g} \mathrm{TE} / 100 \mathrm{~g}$ DW(“TN"). Whereas, at the ripe stage the free radical-scavenging activity exhibited the lowest levels that varied from $11.47 \pm 0.48 \mathrm{~g}$ TE/100 g DW (“GB”) to $4.56 \pm 0.45 \mathrm{~g} \mathrm{TE} / 100 \mathrm{~g} \mathrm{DW}$ (“TN”).

\section{Discussions}

Previous published data were revealed that carob pods contained considerable amounts of minerals $(2,13)$, important levels of bioactive compounds (12) and was considered as interesting source of carbohydrates $(1,11)$ as well antioxidant principles (12). However, its valuation is mainly depends on their nutritional and functional compounds which are accumulated and degraded during ripening process $(3,12)$. Indeed, the users are always looking for a well-finished quality to satisfy their needs, maybe it can be the astringency, the sweetness or the abundance of nutritive or functional principles-(24). In this term the present investigation focused to determine the changes in a significant range of photochemical compounds of carob pods as influenced by ripening stages and provenance in order to extend the use of carob pods.

The storage conditions was correlated with water content (25) which affected the sensorial parameters and nutritional quality of fruits. However, the decline pattern of moisture recorded by selected provenances was necessary to maintain the nutritional quality of mature fruits during storage. This behavior would also enhance the percentage of solid compounds which explains the hardness character of mature pulps. Therefore, we could guess that the "SK" and "GB" provenances are characterized by the hardest texture and predict they will maintain its sensorial and nutritional properties for a longer period. Therefore, fruit that contain considerable amounts of mineral elements could be found as a good source of mineral supplement in food diets. However, several investigation reported the mineral composition of the pods $(2,13,26)$, but no available information about its variation during ripening.

It was seen that ash and mineral elements contents revealed a downward trend, which was related to the mobility of mineral elements in phloem from pods to another parts of fruits such as seeds which are known by their highest ash content $(2,27,28)$. Their participation in various metabolic pathways justify also this behavior (29). In our investigation, the changes that were produced during ripening conferred significant mineral yields at the end of maturity, but it remains lower than those record- ed by the wild and grafted pods harvested from Antalya (2). Besides, it was shown that the ash and minerals contents are susceptible to climate and environmental change which introduced by geographical origin variation. This behavior might be explained by the fact that the transfer of mineral salts in the phloem depends mainly on the environment where the carob trees are grown and the plant state (28). Indeed, the minerals convey from the soil to the plant depends on their availability in the soil, the presence of water and its quality that promotes their absorption and affects the roots state; growth and activity (30). Moreover, the sugars confer particular organoleptic properties and defines the commercial value for carob pods (24). Again, the sugars contents were reported previously by Ayaz et al.(26), Biner et al.(1) and Vekiari et al.(11), who revealed that sucrose, fructose and glucose were the main sugars qualified and quantified in carob pods. The same results are displayed in our investigation. The considerable influence of ripening stage was also demonstrated by others investigations $(11,32)$, where it was indicated that glucose and fructose content showed a decreasing trend during ripening of carob pods, since it would mainly contribute to the biosynthesis of polysaccharides and might be involved in certain physical and chemical reactions $(33,34)$. During fruit development, the sucrose content exhibited an increment which was in agreement with the evolutional pattern exhibited by carob pods harvested from Turkey (11). This behavior is mainly controlled by the profile of sucrose phosphate synthase (SPS) activity. Indeed, Schaffer et al.(35) and Gomez et al. (36) revealed, during ripening of melon and papaya, a synchronization between increased SPS activity and sucrose accumulation. During maturation, the fruits receive a regular portion of sucrose from photosynthesis which occurs in the leaves and found to be the main biosynthesis pathway of sucrose (36). The accumulation of sucrose could be also explained by the breakdown of the different carbohydrates present in the other organs of the plant (15). Indeed, Hubbard et al. (37) found that partial decomposition of stachyose in leaves is relatively related to sucrose synthesis. Schaffer et al. (35) detected significant amounts of raffinose and stachyose, which can be transported to the fruit, while sucrose is the only polysaccharide present in the melon.

Besides, the sucrose was found as a discriminant factor between provenances, since it was present as the predominant sugar in the mature stage and its concentrations varied significantly between the provenances. This variation was generally due to genetic and environmental exhibition that could modify the activity of the enzymes involved in biosynthesis and decomposition of sugars including sucrose phosphate synthase and acid invertase $(15,37)$. Moreover, in sensorial and nutritional term the mature pods obtained from "KE" would be the most appreciated by consumers as a delicious and energetic fruit, since it revealed the highest sugar content. It deserves an industrial development to enhance the yield of syrup, juice and carob flour preparation as well bioethanol production.

The polyphenols were the most important secondary metabolites and considered as an interesting compound define the 
nutritional and functional fruits quality $(12,38)$. As the primary metabolites, this groups of molecules recorded important variation during ripening(12), since some authors revealed that during ripening of fruits the evolutionary behavior of secondary metabolisms was highly associated with that of primary ones $(28,39)$. Indeed, the biosynthesis and biodegradation of these latest would generate the abundance or the deficiency of substrates which contribute to the biosynthesis of secondary metabolisms. The analysis of carob TP content showed a gradual decrease during ripening to attain the lowest levels at the end of the maturity. However, they remain higher than those demonstrated by El-sherif et al. (40) and Benchikh et al. (12) whose investigated the ethanolic extract (186.07 mg GAE/ 100 g) and aqueous extract of carob pods (1.35 g GAE/ $100 \mathrm{~g} \mathrm{DW})$. This significant variation could be related to the difference of harvest date, climatic and geographical conditions, the methods of extraction, the solvent polarity and genetic characters (12).

The observed progressive patterns were in agreement with the results reported by Benchikh et al.(12) and Howard et al.(41) whose studied respectively carob pods harvested from Algeria and pepper fruits. Once more, this tendency was probably explained by the activation of polyphenol oxidase (PPO) which contribute to the oxidation of phenolic compounds (42). The changes in their solubility in water might justify our observations. In fact, at the beginning of maturity the phenolics compounds exhibited weak polar character and then their condensation during ripening coffered an important polarity $(12,43)$. Their participation in enzymatic browning confirm also this pattern. As for condensed tannins, its downward trend is crucial to reduce the severity of astringent taste (39). The "GB" provenance is the most profitable for the users who's looking for the curative properties, since the flavonoids compounds were the main controller of the anti-inflammatory, anti-allergic and anti-cancer activities. The high temperatures in the southern regions might justify these important flavonoids amounts, since it was shown that the lower temperature prevent the accumulation of flavonoids compounds (44). Besides, the rainfall reduced the total phenolic levels (45), therefore the "TN" provenance which grown in northern areas that probably present the highest rainfall level, showed the lowest phenolics compounds content. Moreover, the yields of the dry extracts obtained from studied provenances showed upward tendency during ripening to attain the highest amounts at the ripe stage, which were close to those found by Roseiroet al. (46) for aqueous extract of mature carob pods (53.17\%). This behavior seems reasonable since throughout ripening the percentage of solid compounds showed an increasing trend.

The antioxidant capacity followed the same change pattern shown by the bioactive compounds content which confirms the high correlation between the amounts of bioactive compounds and antioxidant capacity assessed by DPPH test $(28,47)$. The same results were reported by Benchikh et al. (12) whose investigated carob fruits harvest from Algeria. Furthermore, the origin effect was also showed for all determined parameters, since each provenance has its each provenance has its own climatic, geographical and pedological natures, such as rainfall levels, sun exposure and temperature that affect the biosynthesis of phenolic compounds. The interesting content of bioactive compounds and the considerable antioxidant capacity confirmed the great nutritional and functional potential of this fruits, notably at the unripe stage and especially to "GB" provenance.

\section{Conclusions}

Our findings showed that biochemical composition and organoleptic properties were considerably influenced by the ripening and the geographic origin. However, in the studied provenances, ripening was accompanied by a remarkable regression in phenolic compounds correlated with downtrend of antioxidant activity, an interesting decrease in minerals content and a considerable accumulation of sucrose. The behavior exhibited by each component justified the fact that carob fruit could be used largely in all ripening stages depending in targeted nutrient. Therefore, the green carob pods were considered as an interesting source of natural antioxidant for pharmaceutical uses, while the mature pods should be selected as a good sugar source for juice extraction, cacao substitution and bioethanol production.

\section{Conflict of Interest}

The authors declare that they have no conflicts of interest.

\section{Ethical Compliance}

This article does not contain any studies involving human participants or animals performed by any of the authors.

\section{References}

1. Biner B, Gubbuk H, Karhan M, Aksu M, Pekmezci M. Sugar profiles of the pods of cultivated and wild types of carob bean (Ceratonia siliqua L.) in Turkey. Food Chem. 2007;100(4):1453-5.

2. Oziyci HR, Tetik N, Turhan I, Yatmaz E, Ucgun K, Akgul H, et al. Mineral composition of pods and seeds of wild and grafted carob (Ceratonia siliqua L.) fruits. Sci Hortic (Amsterdam) Elsevier B.V. 2014;167:149-52.

3. Rtibi K, Selmi S, Grami D, Amri M, Eto B, El-benna J, et al. Chemical constituents and pharmacological actions of carob pods and leaves (Ceratonia siliqua L.) on the gastrointestinal tract: A review. Vol. 93, Biomedicine and Pharmacotherapy. 2017. p. 522-8.

4. Custódio L, Escapa AL, Fernandes E, Fajardo A, Aligué R, Alberício F, et al. Phytochemical Profile, Antioxidant and Cytotoxic Activities of the Carob Tree (Ceratonia siliqua L.) Germ Flour Extracts. Plant Foods Hum Nutr. 2011;66(1):78-84.

5. Ercan Y, Irfan T, Mustafa K. Optimization of ethanol production from carob pod extract using immobilized Saccharomyces cerevisiae cells in a stirred tank bioreactor. Bioresour Technol. Elsevier Ltd. 2013;135:365-71.

6. Hsouna A Ben, Trigui M, Mansour R Ben, Jarraya RM, Damak M, Jaoua S. Chemical composition, cytotoxicity effect and antimicrobial activity of Ceratonia siliqua essential oil with preservative effects against Listeria inoculated in minced beef meat. Int J Food Microbiol. 2011;148(1):66-72.

7. Dhaouadi K, Belkhir M, Akinocho I, Raboudi F, Pamies D, Barrajón $E$, et al. Sucrose supplementation during traditional carob syrup processing affected its chemical characteristics and biological activities. LWT - Food Sci Technol. 2014;57(1):1-8.

8. Tetik N, Turhan I, Oziyci HR, Gubbuk H, Karhan M, Ercisli S. Physical and chemical characterization of Ceratonia siliqua L. germplasm 
in Turkey. Sci Hortic (Amsterdam). 2011;129(4):583-9.

9. Dakia PA, Wathelet B, Paquot M. Isolation and chemical evaluation of carob (Ceratonia siliqua L.) seed germ. Food Chem. 2007;102(4):1368-74.

10. Almanasrah M, Roseiro LB, Bogel-Lukasik R, Carvalheiro F, Brazinha C, Crespo J, et al. Selective recovery of phenolic compounds and carbohydrates from carob kibbles using water-based extraction. Ind Crops Prod. 2015;70:443-50.

11. Vekiari SA, Georgia, Ozturk M, Görk G. Variation of quality characteristics in Greek and Turkish carob pods during fruit development. In: Procedia - Social and Behavioral Sciences. 2011;19:7505.

12. Benchikh $\mathrm{Y}$, Louaileche $\mathrm{H}$, George B, Merlin A. Changes in bioactive phytochemical content and in vitro antioxidant activity of carob (Ceratonia siliqua L.) as influenced by fruit ripening. Ind Crops Prod. 2014;60:298-303.

13. Özcan MM, Arslan D, Gökçalik H. Some compositional properties and mineral contents of carob (Ceratonia siliqua) fruit, flour and syrup. Int J Food Sci Nutr. 2007;58(8):652-8.

14. Oziyci HR, Tetik N, Turhan I, Yatmaz E, Ucgun K, Akgul H, et al. Mineral composition of pods and seeds of wild and grafted carob (Ceratonia siliqua L.) fruits. Sci Hortic (Amsterdam). 2014;167:14952.

15. Villanueva MJ, Tenorio MD, Esteban MA, Mendoza MC. Compositional changes during ripening of two cultivars of muskmelon fruits. Food Chem. 2004;87(2):179-85.

16. Custódio L, Fernandes E, Escapa AL, Fajardo A, Aligué R, Alberício $\mathrm{F}$, et al. Antioxidant and cytotoxic activities of carob tree fruit pulps are strongly influenced by gender and cultivar. J Agric Food Chem. 2011;59(13):7005-12.

17. Sebai H, Souli A, Chehimi L, Rtibi K, Amri M. In vitro and in vivo antioxidant properties of Tunisian carob ( Ceratonia siliqua $\mathrm{L}$.). 2013;7(2):85-90.

18. Avallone R, Plessi M, Baraldi M, Monzani A. Determination of chemical composition of carob (Ceratonia siliqua): Protein, fat, carbohydrates, and tannins. J Food Compos Anal. 1997;10(2):166-72.

19. Elfalleh W, Nasri N, Marzougui N, Thabti I, Yahya Y, Lachiheb B, et al. Physico-chemical properties and DPPH-ABTS scavenging activity of some local pomegranate ( Punica granatum ) ecotypes. Int. JJ.Food Sci. Nutri. 2009;60: 197-210.

20. Chaira N, Mrabet A, Ferchichi ALI. Evaluation of antioxidant activity, phenolics, sugar and mineral contents in date palm fruits. J Food Biochemistry. 2009;33:390-403.

21. Assadi I, Elfalleh W, Benabderrahim MA, Hannachi $H$, Chaalen W, Ferchichi A. Nutritional Quality and Antioxidant Capacity of a Combination of Pomegranate and Date Juices. Int J Fruit Sci. 2018;1-15.

22. Bahorun T, Gressier B, Trotin F, Brunet C, Dine T, Luyckx M, et al. Oxygen species scavenging activity of phenolic extracts from hawthorn fresh plant organs and pharmaceutical preparations. Arzneimittelforschung. 1996;46(11):1086-9.

23. Mustapha K, Nacéra M, Souhila M. Etude de l'extraction des composés phénoliques de différentes parties de la fleur d'artichaut (Cynarascolymus L.). Nat \&Technologie. 2013;B:35-40.

24. El Batal H, Hasib A, Ouatmane A, Dehbi F, Jaouad A, Boulli A Sugar composition and yield of syrup production from the pulp of Moroccan carob pods (Ceratonia siliqua L.). Arab J Chem. 2016;9:S955-9.

25. Milanez JT, Neves LC, Colombo RC, Shahab M, Roberto SR. Bioactive compounds and antioxidant activity of buriti fruits, during the postharvest, harvested at different ripening stages. Sci Hortic (Amsterdam). 2018;227:10-21.

26. Ayaz FA, Torun H, Ayaz S, Correia PJ, Alaiz M, Sanz C, et al. Determination of chemical composition of anatolian carob pod (Ceratonia siliqua L.): Sugars, amino and organic acids, minerals and phenolic compounds. J Food Qual. 2007;30(6):1040-55.

27. Adeyemi, O. S., Oladiji a. T. Compositional changes in banana ( Musa ssp .) fruits during ripening. African J Biotechnol. 2009;8(5):858-9.

28. Seraglio SKT, Schulz M, Nehring P, Della Betta F, Valese AC, Daguer $\mathrm{H}$, et al. Nutritional and bioactive potential of Myrtaceae fruits during ripening. Food Chem. Elsevier Ltd. 2018;239:649-56.

29. El Buluk R., Babiker E., El Tinay A. Changes in sugar, ash and minerals in four guava cultivars during ripening. Plant Foods Hum Nutr. 1996;49:147-54

30. Esteban M., Villanueva M., Lissarrague J. Effect of Irrigation on Changes in Berry Composition of Tempranillo During Maturation. Sugars, Organic Acids, and Mineral Elements. 1999;Am. J. Eno:418-34.

31. Kusumiyati YA, EizoTaira, Koji Wada. Alterations in the morphological, sugar composition, and volatile flavor properties of petai (Parkia speciosa Hassk.) seed during ripening. Food Res Int. 2018;106:647-53.

32. Farag MA, El-Kersh DM, Ehrlich A, Shokry M, El-Seedi $H$, Frolov A et al. Variation in Ceratonia siliqua pod metabolome in context of its different geographical origin, ripening stage and roasting process. Food Chem. Elsevier Ltd. 2019; 283:675-687.

33. Wang Y, Wyllie SG, Leach DN. Chemical Changes during the Development and Ripening of the Fruit of Cucumis melo (Cv. Makdimon). J Agric Food Chem. 1996;44(1):210-6.

34. Bi J, Qinqin Chen XW, Ying L, Xianjun Meng JS. Assessment of sugar content, fatty acids, free amino acids, and volatile pro fi les in jujube fruits at different ripening stages. Food Chem. 2019;270:34452.

35. A.Schaffer A, Aloni B, Fogelman E. Sucrose metabolism and accumulation in developing fruit of Cucumis. Phytochemistry. 1987;26:1883-7.

36. Gomez M, Lajolo F, Cordenunsi B. Evolution of Soluble Sugars During Ripening of Papaya Fruit and its Relation to Sweet Taste. J Food Sci. 2002;67:442-7.

37. Hubbard NL, Huber SC, Pharr DM. Sucrose Phosphate Synthase and Acid Invertase as Determinants of Sucrose Concentration in Developing Muskmelon ( Cucumis melo L .) Fruits1. PlantPhysiol. 1989;91:1527-34.

38. Tetik N, Yüksel E. Ultrasound-assisted extraction of d-pinitol from carob pods using Response Surface Methodology. Ultrason Sonochem. 2014;21(2):860-5.

39. P.Kulkarni A, Mallikarjuna Aradhya S. Chemical changes and antioxidant activity in pomegranate arils during fruit development. Food Chem. 2005;93:319-24.

40. El-sherif $\mathrm{G}$, El-sherif MA, Tolba KH. Extraction and Identification of Natural Antioxidants from Liquorices (Glycyrrhiza glabra) and Carob (Ceratonia siliqua) and Its Application in ElMewled El-Nabawy Sweets (Sesames and Folia). Nature.science. 2011;8:108-15.

41. Howard LR, Talcott ST, Brenes CH, Villalon B. Changes in Phytochemical and Antioxidant Activity of Selected Pepper Cultivars (Capsicum Species) As Influenced by Maturity. J Agric Food Chem. 2000;48:1713-1720.

42. Gull J, Sultana B, Anwar F, Naseer R, Ashraf M AM. Variation in Antioxidant Attributes at Three Ripening Stages of Guava (Psidium guajava L.) Fruit from Different Geographical Regions of Pakistan. molecules. 2012;17:3165-80.

43. Gull J, Sultana B, Anwar F, Naseer R, Ashraf M, Ashrafuzzaman M. Variation in antioxidant attributes at three ripening stages of guava (Psidium guajava L.) fruit from different geographical regions of Pakistan. Molecules. 2012;17(3):3165-80.

44. Reay PF, Lancaster JE. Accumulation of anthocyanins and quercetin glycosides in ' Gala 'and ' Royal Gala 'apple fruit skin with UV-B \pm Visible irradiation : modifying effects of fruit maturity , fruit side , and temperature. 2001;90:57-68.

45. Lima VLAG, Mélo EA, Maciel MIS, Prazeres FG, Musser RS, Lima DES. Total phenolic and carotenoid contents in acerola genotypes harvested at three ripening stages. Food Chem. 2005;90(4):565-8.

46. Roseiro LB, Duarte LC, Oliveira DL, Roque R, Bernardo-Gil MG, Martins $\mathrm{Al}$, et al. Supercritical, ultrasound and conventional extracts from carob (Ceratonia siliqua L.) biomass: Effect on the phenolic profile and antiproliferative activity. Ind Crops Prod. 2013;47:132-

47. Suzie Zozio ASGC, Didier Mbéguié-A-Mbéguié, SylvieRavion. Changes in antioxidant activity during the ripening of Jujube (ZiziphusmauritianaLamk). Food Chem. 2014;150:448-56. 